\title{
Influence of Recombination Centers on the Phase Portraits in Nanosized Semiconductor Films
}

\author{
Gafur Gulyamov $^{1}$, Abdurasul G. Gulyamov², Feruza R. Muhitdinova ${ }^{3}$ \\ ${ }^{1}$ Namangan Engineering-Pedagogical Institute, Namangan, Uzbekistan \\ ${ }^{2}$ Physico-technical Institute, Academy of Sciences of Uzbekistan, Tashkent, Uzbekistan \\ ${ }^{3}$ Namangan State University, Namangan, Uzbekistan \\ Email: gulyamov1949@mail.ru
}

How to cite this paper: Gulyamov, G., Gulyamov, A.G. and Muhitdinova, F.R. (2016) Influence of Recombination Centers on the Phase Portraits in Nanosized Semiconductor Films. Journal of Modern Physics, 7, 16611667.

http://dx.doi.org/10.4236/jmp.2016.713151

Received: July 12, 2016

Accepted: September 11, 2016

Published: September 14, 2016

Copyright $\odot 2016$ by authors and Scientific Research Publishing Inc. This work is licensed under the Creative Commons Attribution International License (CC BY 4.0). http://creativecommons.org/licenses/by/4.0/

\begin{abstract}
Influence of recombination centers' changes on the form of phase portraits has been studied. It has been shown that the shape of the phase portraits depends on the concentration of semiconductor materials' recombination centers.
\end{abstract}

\section{Keywords}

Recombination Centers, Phase Portrait, Generation of Charge Carriers, Recombination of Charge Carriers, Forbidden Zone, Absorption Coefficient

\section{Introduction}

Operation recombination processes allow to control the number of excess charge carriOpen Access portance at the production of semiconductor devices. There are many types of recombination such as linear recombination and quadratic one, recombination through recombination centers and radiative recombination [1]-[3]. When recombination goes through recombination centers, the transition of the charge carriers from a free state to a bound one is independent of the presence of excess charge carriers of opposite sign. This means that there is no direct connection of an electron and the hole, that is, to complete the act first capturing one sign carrier by trap takes place and then capturing of the opposite sign does. There are many internal and external factors contributing to the growth and reduction of recombination centers. Changing the number of recombination centers may be undesirable for semiconductor devices and can disable them. In order to prevent interruption in the semiconductor devices work, it is necessary to conduct diagnostic of generation-recombination processes, and if it is necessary, re- 
place them. The process of generation and recombination is similar to the oscillatory process, since the increase and the decrease of concentration of charge carriers occur periodically. For analysis of oscillatory processes, phase portraits $(\mathrm{PhP})$ are used effectively, because they give the most complete picture of what is happening. In [4] using $\mathrm{PhP}$ the influence of frequency of variable deformation on the concentration of charge carriers in semiconductor illuminated by its forbidden zone's light, it is shown that the frequency of the variable deformation has a strong influence on the shape of the $\mathrm{PhP}$. However, in [4] influence of recombination centers in the form of $\mathrm{PhP}$ is not investigated. In this paper, we investigate influence of the change of recombination centers in the semiconductor by $\mathrm{PhP}$.

\section{The Continuity Equation Taking into Account the Combined Effects of Light and Variable All-Round Deformation}

The continuity equation expressing the change of concentration of charge carriers is described by the following expression

$$
\frac{\partial n_{e}}{\partial t}=g-r+\frac{1}{q} \nabla \boldsymbol{I}_{n}
$$

where $g$-rate of generation of charge carriers, $r$-rate of recombination of charge carriers, $q$-charge of electron, $\boldsymbol{I}_{n}$-the current density of electrons. In uniform sample, the continuity equation has the following form

$$
\frac{\partial n_{e}}{\partial t}=g-r
$$

when illuminated by its forbidden zone's light semiconductor becomes sensitive to external influences change of the absorption coefficient contributes to that [5]. At radiation generation is expressed by the following expression $g_{s}=\frac{\alpha I}{h v}$. Here, $\alpha$-absorption coefficient, $I$-intensity of the light, $h$-Planck's constant, $v$-the frequency of the light. With the express permission of the transition frequency dependence of the absorption coefficient is $\alpha=A\left(h v-E_{g}\right)^{1 / 2}=A h^{1 / 2}\left(v-v_{m}\right)^{1 / 2}$ where $v_{m}$-the frequency of its forbidden zone's light [1] [3] [6] $A$-certain coefficient which is defined by the following expression $A=\frac{q^{2}\left(2 m_{n p}^{*}\right)^{3 / 2}}{n c h^{2} m_{e}^{*}}$ for direct allowed transitions $A \approx 2 \times 10^{4} \quad[6]$, here $m_{n p}^{*}=\frac{m_{h}^{*} m_{e}^{*}}{m_{h}^{*}+m_{e}^{*}}$-is the reduced effective mass, $m_{e}^{*}, m_{h}^{*}$-the effective mass of electrons and holes, respectively $n$-the index of refraction of the light, $c$-velocity of the light.

Let's consider the case when deformation of all-round strain effects on the semiconductor. When the deformation of band gap changes as follows [7]-[9] $E_{g}=E_{g}(0)-\Xi \varepsilon$, here $\Xi$-the constant of the deformation potential, $\varepsilon$-relative deformation. In the case $E_{g}(0)=h v$, then if $v=v_{m},\left(h v-E_{g}(0)+\Xi \varepsilon\right)^{1 / 2}=(\Xi \varepsilon)^{1 / 2}$ the absorption coefficient becomes $\alpha=A(\Xi \varepsilon)^{1 / 2}$. If the deformation changes periodically $\varepsilon=\varepsilon_{0}\left(1+\sin \omega_{d} t\right)$, whereas $\alpha=A\left(\Xi \varepsilon_{0}\left(1+\sin \omega_{d} t\right)\right)^{1 / 2}$ here $\omega_{d}$ frequency of variable deformation, here 
generation at light $g_{s}=\frac{A\left(\Xi \varepsilon_{0}\left(1+\sin \omega_{d} t\right)\right)^{1 / 2} I}{h v}$. Equation (2) for this case is as follows:

$$
\frac{\partial n}{\partial t}=g_{0}\left(1+\sin \omega_{d} t\right)^{1 / 2}-r, \quad g_{0}=\frac{A\left(\Xi \varepsilon_{0}\right)^{1 / 2} I}{h v}
$$

At direct unpermitted transition frequency dependence of the absorption coefficient $\alpha=B\left(h v-E_{g}\right)^{3 / 2}=B h^{3 / 2}\left(h-h_{m}\right)^{3 / 2}$ where $v_{m}$-frequency of its forbidden zone's light, $B$-coefficient that is defined by the following expression $B=\frac{4}{3} \frac{q^{2}\left(2 m_{n p}^{*}\right)^{5 / 2}}{n c h^{2} m_{e}^{*} m_{h}^{*} h v}, \quad B \approx 1.3 \times 10^{4}$ [6].

Equation (2) for direct unpermitted transition will be as follows:

$$
\frac{\partial n}{\partial t}=g_{0}\left(1+\sin \omega_{d} t\right)^{3 / 2}-r, g_{0}=\frac{B\left(\Xi \varepsilon_{0}\right)^{3 / 2} I}{h v}
$$

Taking into account the permitted and unpermitted direct transition continuity equation takes the form

$$
\frac{\partial n}{\partial t}=\frac{A\left(\Xi \varepsilon_{0}\right)^{1 / 2} I}{h v}\left(1+\sin \omega_{d} t\right)^{1 / 2}+\frac{B\left(\Xi \varepsilon_{0}\right)^{3 / 2} I}{h v}\left(1+\sin \omega_{d} t\right)^{3 / 2}-r
$$

Let's consider the case where the recombination through recombination centers takes place. According to statistics of the Shockley-Read, the rate of recombination is described by the expression: $r=N_{t} \frac{c_{n} c_{p}\left(p n-n_{i}^{2}\right)}{c_{n}\left(n+n_{1}\right)+c_{p}\left(p+p_{1}\right)}$ Here, $N_{t}$-the concentration of recombination centers, $c_{n}$ and $c_{p}$ coefficients capture electrons and holes, respectively [1] [3]. In this case, the continuity equation is

$$
\begin{aligned}
& \frac{\partial n}{\partial t}=\frac{A\left(\Xi \varepsilon_{0}\right)^{1 / 2} I}{\hbar \omega}\left(1+\sin \omega_{d} t\right)^{1 / 2}+\frac{B\left(\Xi \varepsilon_{0}\right)^{3 / 2} I}{\hbar \omega}\left(1+\sin \omega_{d} t\right)^{3 / 2} \\
& -N_{t} \frac{c_{n} c_{p}\left(p n-n_{i}^{2}\right)}{c_{n}\left(n+n_{1}\right)+c_{p}\left(p+p_{1}\right)}
\end{aligned}
$$

\section{Analysis of Phase Portraits}

Let's consider the effect of deformation on a illuminated semiconductor. Let's assume the following values: the temperature $T=300 \mathrm{~K}$, the band gap $E g=1.1 \mathrm{eV}$, the relative deformation $\varepsilon=10^{-6}$, the deformation potential's constant is $\Xi=11.4 \mathrm{eV}$, lightl's intensity $I=10^{18} \mathrm{~cm}^{-2} \cdot \mathrm{sek}^{-1}$, the effective mass off electron $m_{n}^{*}=0.4 \cdot m_{0}$, were $m_{0}$-mass of free electron, the effective mass of holes $m_{p}^{*}=0.541 \cdot m_{0}$, the coefficient of direct allowed transitions $A=2 \times 10^{4}$ [6], the coefficient of unpermitted direct transition $B=$ $1.3 \times 10^{4}$ [6], the concentration of recombination centers $N_{t}=2 \times 10^{13} \mathrm{~cm}^{-3}$, the electron capture coefficient $c_{n}=4.4 \times 10^{-10} \mathrm{~cm}^{3} / \mathrm{sek}$, hole capture coefficient $c_{p}=6.2 \times 10^{-9}$ $\mathrm{cm}^{3} / \mathrm{sek}$, the intrinsic concentration $n_{i}=2.2 \times 10^{13} \mathrm{~cm}^{-3}$ [6] [10] [11]. Let the frequency of the variable all-round compression is $\omega_{d}=250 \mathrm{~Hz}$. Let's consider one period of variable strain. Let's consider the phase portrait in 0.3 seconds after the start of a periodic 
deformation. The beginning of counting out is $t=0.3 \mathrm{sek}$, because by this time the carrier concentration becomes the stable value, $n \approx 6 \times 10^{15} \mathrm{~cm}^{-3}$ in which will returns periodically at the process of variable deformation of the sample (see Figure 1 and Figure 2 , point 1). As the deformation increases of the carrier concentration will increase and reach its peak $n \approx 1 \times 10^{16} \mathrm{~cm}^{-3}$ at point 2 as shown in Figure 1 and Figure 2. After this, decrease of the strain begins and the concentration at the point 3 has again significance $n \approx 6 \times 10^{15} \mathrm{~cm}^{-3}$. Further, when the strain is completely takes off the concentration takes minimum value $n \approx 10^{15} \mathrm{~cm}^{-3}$ at point 4 . Upon completion of the period of hydrostatic compression at point 5 the concentration again returns to its primary value $n \approx 6 \times 10^{15} \mathrm{~cm}^{-3}$. This process will continue in this way until all parameters of the oscillatory system remain constant, and the phase portrait will take the form of a closed loop. $n \approx 10^{15} \mathrm{~cm}^{-3}$

At prolonged effect of the strain, especially if the deformation is variable there is the probability of increase of recombination centers. The appearance of new defects and structural changes in the semiconductor caused by fatigue and wear material promote it. Let's consider the effect of changes in the concentration of recombination centers in the form of phase portraits. Let the concentration of recombination centers grows increases from $2 \times 10^{13} \mathrm{~cm}^{-3}$ to $8 \times 10^{13} \mathrm{~cm}^{-3}$, in this case the phase portrait will not be in the form of a closed loop, but it will curl into a spiral form (see Figure 3 ). The maximum value of the carrier concentration will be $n \approx 1.6 \times 10^{16} \mathrm{~cm}^{-3}$ when $N_{t}=2 \times 10^{13} \mathrm{~cm}^{-3}$, and at $N_{t}=8 \times 10^{13} \mathrm{~cm}^{-3}$ the maximum concentration of charge carriers will be

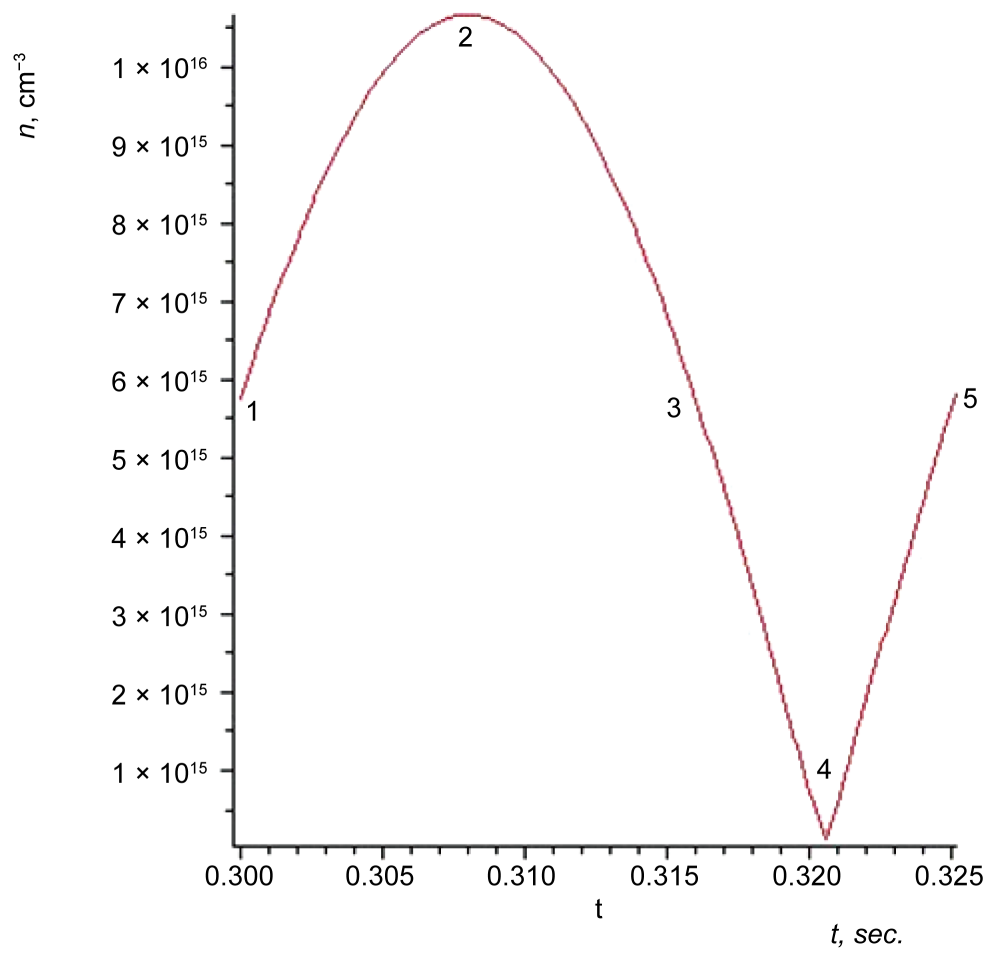

Figure 1. Dynamics of changes of the concentration of charge the carriers in the period of variable strain. 


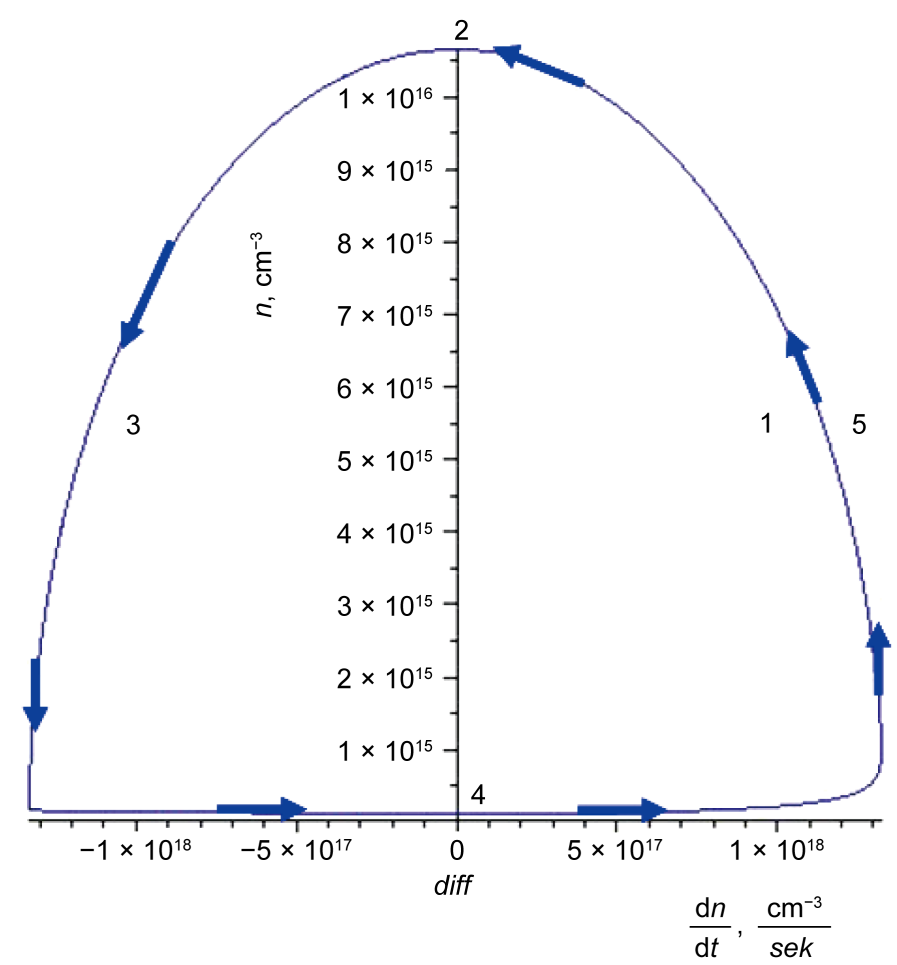

Figure 2. Phase portrait of the concentration $(n)$ of charge carriers versus the rate of change of the charge carrier concentration $(\mathrm{d} n / \mathrm{d} t)$.

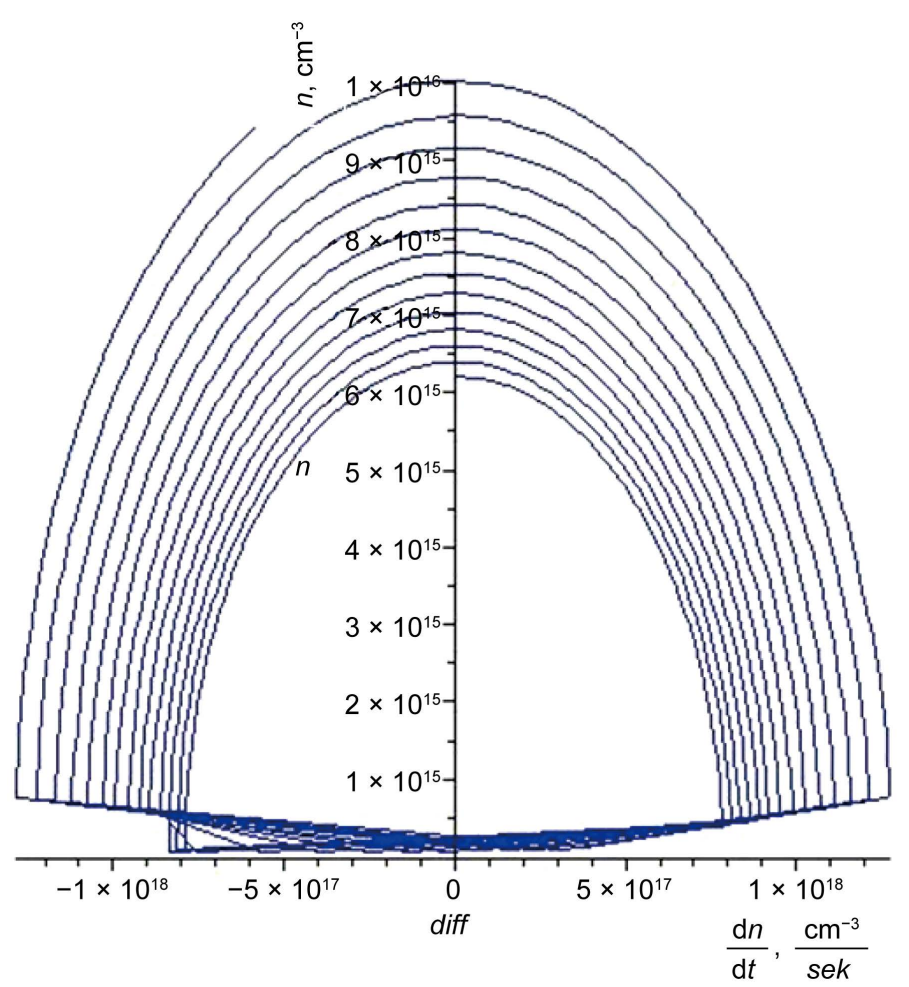

Figure 3. Phase portrait $n$ versus $\mathrm{d} n / \mathrm{d} t$, for the case when the concentration of recombination centers variers from $N_{t}=2 \times 10^{13} \mathrm{~cm}^{-3}$ to $N_{t}=8 \times 10^{13} \mathrm{~cm}^{-3}$. 
$n \approx 6 \times 10^{15} \mathrm{~cm}^{-3}$. Such decrease of the concentration of charge carriers can be explained by the fact that the generation changes in a constant range, and the range of the recombination increases with time.

Figure 4 shows the phase portrait for the case when the concentration of recombination centers decreases from $2 \times 10^{13} \mathrm{~cm}^{-3}$ to $2 \times 10^{12} \mathrm{~cm}^{-3}$. In this case, when $N_{t}=2 \times 10^{13} \mathrm{~cm}^{-3}$ the maximum concentration is $n \approx 1.6 \times 10^{16} \mathrm{~cm}^{-3}$, and when $N_{t}=2 \times 10^{12} \mathrm{~cm}^{-3}$ the maximum concentration is $n \approx 2.9 \times 10^{16} \mathrm{~cm}^{-3}$. Increasing concentration of charge carriers is caused by that the range of the recombination term $R$ in the Equation (2) decreases but the range of generation term $g$ remains constant.

Consideration of the phase picture's transformation at the change of the system parameters is very important for understanding the physical processes in the system. Looking at the "phase portrait" under certain given values of the parameters it is possible to imagine all the possible movements in the system for any initial values. While you observe the modification in the picture at the change of the parameters, you represent all advances that the given physical system can have for all possible values of the parameters. For example, the location and nature of the singular points on the phase plane make possible to do a number of conclusions about the processes in the system.

Research of generational process by phase portraits method has practical importance because the phase portraits give the most complete picture of what is happening in the semiconductor. This allows make diagnostics of semiconductor devices and to replace them timely for preventing interruption of their work.

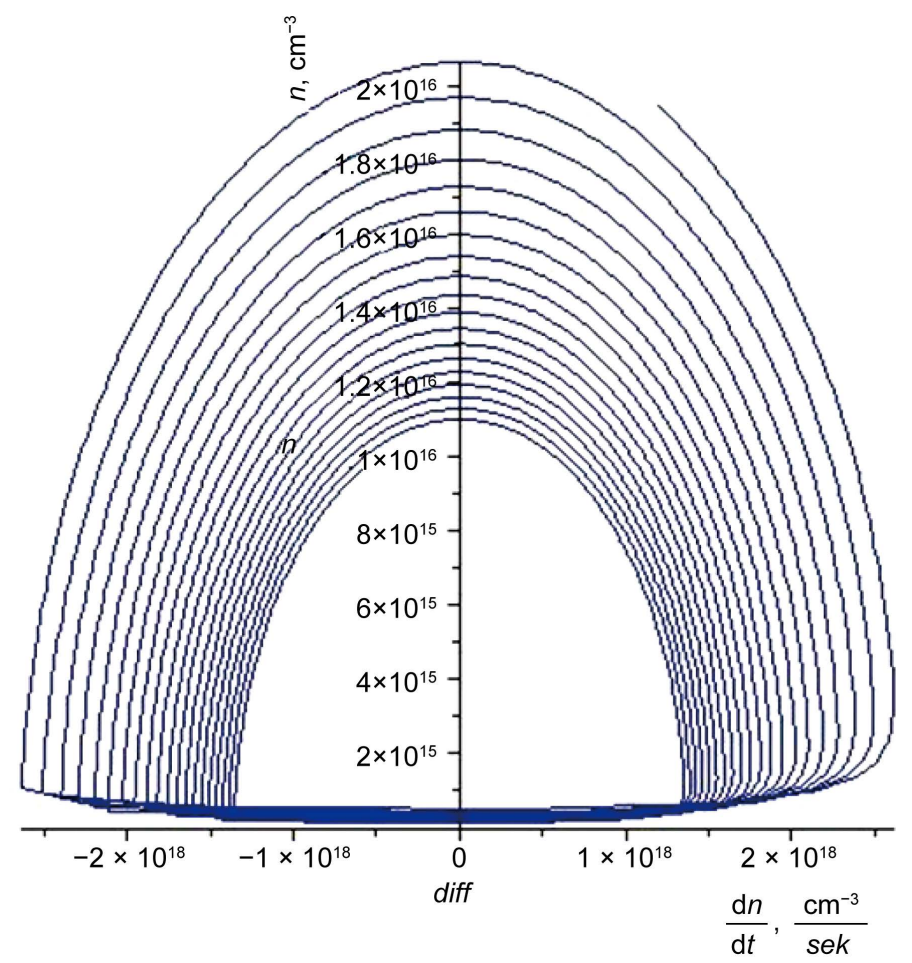

Figure 4. Phase portrait $n$ versus $\mathrm{d} n / \mathrm{d} t$, for the case when the concentration of recombination centers variers from $N_{t}=2 \times 10^{13} \mathrm{~cm}^{-3}$ to $N_{t}=2 \times 10^{12} \mathrm{~cm}^{-3}$. 


\section{Conclusions}

Thus, the study of generation-recombination processes on the basis of the phase portraits allows us to make the following conclusions:

- Increasing recombination centers in the semiconductor causes decrease of carrier concentration, and the phase trajectory rolls spirally toward the lowest values, both on the axis $n$ and on the axis $\mathrm{d} n / \mathrm{d} t$.

- Decreasing of recombination centers in the semiconductor causes with increasing carrier concentration, and the phase trajectory is set in a spiral towards the largest values as along the axis $n$ as along the axis $\mathrm{d} n / \mathrm{d} t$.

- The phase portraits permitted to conduct diagnostics of semiconductor devices

\section{Acknowledgements}

This work was supported by government grants of Uzbekistan F2-OT-O-15494 "Improving the efficiency of the radiators, solar cells and other optoelectronic devices based on quantum dot research and hetero nanostructure exciton and bipolaron transport phenomena", F2-FA-0-97004 "Dynamics of solitons in ultracold quantum gases, and optical systems and synergistic processes in multicomponent semiconductors" and F2-21 "Mathematical modelling of the determination of the density of surface states at the semiconductor-insulator".

\section{References}

[1] Bonch-Bruevich, V.L. and Kalashnikov, S.G. (1977) Semiconductor Physics. Nauka, Moscow, 674 p.

[2] Abakumov, V.N., Perel, V.I. and Yassievich, I.N. (1991) Nonradiative Ecombination in Semiconductors. In: Agranovich, V.M. and Maradudin, A.A., Eds., Modern Problems in Condensed Matter Sciences, North-Holland, Amsterdam, 376 p.

[3] Kireev, P.S. (1975) Semiconductor Physics. Vishaya Shkola, Moscow, 584 p.

[4] Gulyamov, G., Gulyamov, A.G. Ergashev, A.Q. and Abdulazizov, B.T. (2015) Journal of Modern Physics, 6, 1921-1926. http://dx.doi.org/10.4236/jmp.2015.613197

[5] Gulyamov, G. and Gulyamov, A.G. (2015) Semiconductors, 49, 819-822. http://dx.doi.org/10.1134/S1063782615060111

[6] Pankove, J.I. (1971) Optical Processes in Semiconductors. Prentice-Hall, Inc., Englewood Cliffs, $457 \mathrm{p}$.

[7] Polyakova, A.L. (1979) Deformation of Semiconductors and Semiconductor Devices. Energy, Moscow, 168 p.

[8] Gulyamov, A.G. (2015) World Journal of Condensed Matter Physics, 5, 60-65. http://dx.doi.org/10.4236/wjcmp.2015.52009

[9] Bir, G.L. and Pikus, G.E. (1972) Symmetry and Strain-Induced Effects in Semiconductors. Nauka, Moscow, 584 p.

[10] Baranskiy, P.I., Klotchkov, V.P. and Potykevich, I.V. (1975) Semiconductor Electronics. Naukova Dumka, Kiev.: 704 p.

[11] Bonch-Bruevich, V.L., Zvyagin, I.P., Karpenko, I.V. and Mironov, A.G. (1987) Problems in Physics of Semiconductors. Nauka, Moscow, $144 \mathrm{p}$. 
Submit or recommend next manuscript to SCIRP and we will provide best service for you:

Accepting pre-submission inquiries through Email, Facebook, LinkedIn, Twitter, etc. A wide selection of journals (inclusive of 9 subjects, more than 200 journals)

Providing 24-hour high-quality service

User-friendly online submission system

Fair and swift peer-review system

Efficient typesetting and proofreading procedure

Display of the result of downloads and visits, as well as the number of cited articles

Maximum dissemination of your research work

Submit your manuscript at: http://papersubmission.scirp.org/

Or contact jmp@scirp.org 